\title{
Provably Secure Single Sign-on Scheme in Distributed Systems and Networks
}

\author{
Jiangshan Yu, Guilin Wang, and Yi Mu \\ Center for Computer and Information Security Research \\ School of Computer Science and Software Engineering \\ University of Wollongong, Australia \\ Email: \{jy898,guilin,ymu\}@uow.edu.au
}

\begin{abstract}
Distributed systems and networks have been adopted by telecommunications, remote educations, businesses, armies and governments. A widely applied technique for distributed systems and networks is the single sign-on (SSO) which enables a user to use a unitary secure credential (or token) to access multiple computers and systems where he/she has access permissions. However, most existing SSO schemes have not been formally proved to satisfy credential privacy and soundness of credential based authentication. To overcome this drawback, we formalise the security model of single sign-on scheme with authenticated key exchange. Specially, we point out the difference between soundness and credential privacy, and define them together in one definition. Also, we propose a provably secure single sign-on authentication scheme, which satisfies soundness, preserves credential privacy, meets user anonymity, and supports session key exchange. The proposed scheme is very efficient so that it suits for mobile devices in distributed systems and networks.
\end{abstract}

Index Terms-Single sign-on, Distributed systems and networks, Soundness, Authentication, Information security.

\section{INTRODUCTION}

With the wide spreading of distributed computer networks, various network services have gained importance and popularity in recent few years [1][2]. Consequently, user authentication [3] has been widely used in distributed computer networks to identify a legal user who requires accessing network services. To prevent bogus servers, mutual authentication should be considered, and also, a session key establishment is normally required. In addition, user privacy may be desired in distributed computing environments since the information exchanged might be abused by some organizations for marketing purposes [4]. However, designing efficient and secure mutual authentication protocols is challenging in computer networks.

Moreover, with the increasing usage of network services, a user may need to maintain more and more ID/password pairs for accessing different distributed service providers, which impose a burden on users and service providers as well as the communication overhead of computer networks. Single sign-on (SSO) mechanism [5] provides a good remedy to this problem, as it allows a user with a single credential to access multiple service providers. Intuitively, there are three basic security requirements for SSO schemes, namely completeness, soundness and credential privacy [16], [6]. However, to the best of our knowledge soundness has not been formally studied yet and how to preserve both soundness and credential privacy is still a challenge [6].

In 2000, Lee and Chang [7] first proposed an SSO scheme with user anonymity. Later, $\mathrm{Wu}$ and $\mathrm{Hsu}$ [8] pointed out that Lee-Chang scheme suffers from masquerading attack and identity disclosure attack. Meanwhile, Yang et al. [9] showed that $\mathrm{Wu}-\mathrm{Hsu}$ scheme can not preserve credential privacy either since a malicious service provider can recover users' credentials, and then proposed an improvement to overcome this limitation. In 2006, however, Mangipudi and Katti [10] pointed out that Yang et al.'s scheme is insecure against DoS (Deniable of Service) attack and presented a new scheme. In 2009, Hsu and Chuang [11] demonstrated that both Yang et al. and Mangipudi-Katti schemes have not provided user anonymity since their schemes are vulnerable to identity disclosure attacks. To prevent such attacks, Hsu and Chuang proposed an RSA-based user identification scheme.

Recently, Chang and Lee [12] pointed out that Hsu-Chuang scheme is vulnerable to impersonation attacks and the scheme requires additional time-synchronized mechanisms which has unstable latency in distributed networks. Then, they proposed a user anonymity preserving improvement with high efficiency. The scheme uses random nonce to replace additional timesynchronized mechanism, does not need PKI (Public key infrastructure) for users, and suits for mobile device users. However, the security analysis [6] shows that Chang-Lee scheme fails to provide proper user authentication and to preserve credential privacy since the knowledge proof of user authentication guarantees neither soundness nor credential privacy.

As promoted in [6], it is worthy to overcome the flaws in Chang-Lee scheme to obtain an efficient and provably secure scheme for mobile device users in distributed systems and networks. Moreover, the soundness of credential based authentication should be formalised and the credential privacy should be preserved. Motivated to solve these issues, in this paper we first specify a formal model for SSO with a unified definition to formally specify soundness and credential privacy (Section II). Then, after reviewing Chang-Lee SSO scheme in Section III and Schnorr signature [13] in Section IV, we improve Chang-Lee scheme by exploiting Schnorr signature in Section V due to its simplicity and unforgeability [14], [15], while keep Chang-Lee's session key establishment part 
unchanged. The security of the proposed protocol is discussed in Section VI. Finally, section VII concludes this paper.

\section{FORMAL MODEL}

In this section we present a formal model to define authenticated key exchange single sign-on (AKESSO) scheme and its security requirements. Specially, we list the components (e.g. syntax) of $A K E S S O$, define correctness, describe an adversary model, and formally specify three security properties, including secure credential based user authentication, secure credential based service provider authentication, and session key security.

Definition 1. An authenticated key exchange single signon (AKESSO) scheme comprises a trusted credential provider $T C P$, a group of service providers $P$ and a group of users $U$. It consists of eight algorithms and one protocol: initialization algorithm Init(.), identity generation algorithm IdGen(.), credential generation algorithm $C G e n(\cdot)$, credential verification algorithm $C V$ er $(\cdot)$, user proof generation algorithm $U P G e n(\cdot)$, user proof verification algorithm $U P V \operatorname{Ver}(\cdot)$, service provider proof generation algorithm SPPGen(.), and service provider proof verification algorithm SPPVer(.), and key exchange protocol $\prod$.

1) Init $(\lambda)$ : Taking security parameter $\lambda_{0}\left(\right.$ or $\left.\lambda_{1}\right)$ as input, outputs the public/private key pair $(P K, S K)$ for $T C P$ (or $\left(P K_{j}, S K_{j}\right)$ for $\left.P_{j} \in P\right)$.

2) IdGen $\left(R I_{i}\right)$ : Taking registration information $R I_{i}$ as input, outputs an unique identity $I D_{i}$ for a user $U_{i} \in U$.

3) $C G e n\left(I D_{i}, S K\right)$ : Taking an identity $I D_{i}$ and $T C P$ 's private key $S K$ as input, outputs a credential $C_{i}$ for user $U_{i}$.

4) $C V \operatorname{er}\left(C_{i}, I D_{i}, P K\right)$ : Taking credential $C_{i}$, an identity $I D_{i}$, and TCP's public key $P K$ as input, outputs " 1 " or " 0 " for accepting or rejecting credential $C_{i}$ respectively.

5) UPGen $\left(C_{i}, I D_{i}, P K, M\right)$ : Taking a credential $C_{i}$, an identity $I D_{i}$, TCP's public key $P K$ and a temporal message $M$ generated in a session as input, outputs a user proof up $p_{i}$ showing user $U_{i}$ 's knowledge of credential $C_{i}$

6) UPVer $\left(u p_{i}, I D_{i}, P K, M\right)$ : Taking a user proof $u p_{i}$, an identity $I D_{i}$, TCP's public key $P K$, and a temporal message $M$ generated in a session as input, outputs " 1 " or " 0 " for accepting or rejecting up $p_{i}$ as a valid credential proof w.r.t. identity $I D_{i}$ respectively.

7) $S P P G e n\left(S K_{j}, M^{\prime}\right)$ : Taking service provider $P_{j}$ 's private key $S K_{j}$ and a temporal message $M^{\prime}$ generated in a session as input, outputs a service provider proof $s p_{j}$ showing $P_{j}$ 's knowledge of $S K_{j}$.

8) $S P P V \operatorname{Vr}\left(\operatorname{spp}_{j}, P K_{j}, M^{\prime}\right)$ : Taking a service provider proof $\operatorname{spp}_{j}, P_{j}$ 's public key $P K_{j}$, and a temporal message $M^{\prime}$ generated in a session as input, outputs "I" or "O" for accepting or rejecting $\operatorname{spp}_{j}$ as a valid service provider proof w.r.t. public key $P K_{j}$ respectively.
9) $\prod$ : This is a key exchange protocol run by a user $U_{i}$ with private input $C_{i}$ and a service provider $P_{j}$ with private input $S K_{j}$. After the completion of each protocol instance, $U_{i}$ will output a session key $K_{i j}$ if he/she accepts $P_{j}$. Similarly, after the completion of each protocol instance $P_{j}$ will output a session key $K_{j i}$ if it accepts $U_{i}$. (Ideally, $K_{i j}$ and $K_{j i}$ are expected to be the same value.)

Remark 1. The above definition focuses on public key based AKESSO with non-interactive proofs. It could be extended to support interactive proofs, where $s p_{i}$ and $s s p_{j}$ are generated by interactive protocols run by user $U_{i}$ and service provider $P_{j}$. However, defining symmetric key based AKESSO will be another story, which is out the scope of this paper.

Remark 2. Compared to Han et al.'s formal model given in [16], we require key exchange in AKESSO, and each user does not need to hold a public/private key pair. However, in Han et al.'s definition TCP (called IdP in their paper) is less trusted as it will not be able to impersonate any user: Each user will run a zero knowledge protocol to show that he/she knows the private key corresponding to the public key embedded in his/her credential.

Before formally defining security properties, we naturally require an AKESSO should be correct. Namely, a credential $C_{i}$ generated by the trusted credential provider $T C P$ will be valid, a user proof $u p_{i}$ issued properly by user $u_{i}$ who holds a valid credential $C_{i}$ will be accepted by a service provider $P_{j}$ according to $U P V$ er algorithm, a service provider proof $s p p_{j}$ issued properly by $P_{j}$ will be accepted by user $U_{i}$ according to $S P P V$ er algorithm, and $U_{i}$ and $P_{j}$ will accept each other and output the same session key if they honestly run the key exchange protocol $\prod$. Formally, we define correctness as below.

Definition 2. (Correctness) An AKESSO scheme is called correct if it satisfies all the following conditions:

1) For any $R I_{i}$ and any key pair $(P K, S K)$, if $I D_{i} \leftarrow$ $\operatorname{IdGen}\left(R I_{i}\right)$ and $C_{i} \leftarrow C G e n\left(I D_{i}, S K\right)$, then $C V \operatorname{Ver}\left(C_{i}, I D_{i}, P K\right)=1$.

2) For any $I D_{i}$, any key pair $(P K, S K)$ and any $M$, if $C_{i} \leftarrow C G e n\left(I D_{i}, S K\right)$ and $u p_{i} \leftarrow$ $U P G e n\left(C_{i}, I D_{i}, P K, M\right)$, then UPVer $\left(u p_{i}, I D_{i}\right.$, $P K, M)=1$.

3) For any key pair $\left(P K_{j}, S K_{j}\right)$ and any $M^{\prime}, \quad$ if $\operatorname{spp}_{j} \leftarrow \operatorname{SPPGen}\left(S K_{j}, M^{\prime}\right)$, then $S P P V \operatorname{er}\left(\operatorname{spp}_{j}, P K_{j}, M^{\prime}\right)=1$.

4) For any user $U_{i}$ with valid credential $C_{i}$ and service provider $P_{j}$ with private key $S K_{j}$, if both of them run the key exchange protocol $\prod$ honestly, then they will accept each other and output the same session key, i.e., $K_{i j}=K_{j i}$.

Informally, an AKESSO scheme is secure if all the desired functionalities given in the above definition can be carried out only by the proper entities, i.e., not by attackers who 
are allowed to access all possible resources in a rigorously specified adversary model. In fact, we shall define security of SSO authentication which corresponds to items 1) to 3), and session key privacy which corresponds to item 4).

To further define these security properties, we specify the adversary model as follows: Let $\prod_{T C P}$ be the trusted authority oracle with its key pair $(S K, P K), \prod_{U, P}^{i}$ be the user oracle simulating a set of all registered users, interacting with the service provider oracle in session $i$, and $\prod_{P, U}^{j}$ be the service provider oracle simulating a set of all registered service providers, interacting with the user oracle in the session $j$. A probabilistic polynomial time (PPT) adversary $A$ can ask the following oracle queries.

1) $\mathcal{O}_{1}$ : Register $\left(\prod, U\right)$ - Upon receiving this query, the $\prod_{T C P}$ will run $\operatorname{IdGen}\left(R I_{A_{i}}\right)$ and $C G e n\left(I D_{A_{i}}, S K\right)$ algorithms, and output a new user identity $I D_{A_{i}}$ with corresponding credential $C_{A_{i}}$ to $A$ who can verify the credential by running $C V \operatorname{er}(\cdot)$.

2) $\mathcal{O}_{2}$ : Register $\left(\prod, P\right)$ - Upon receiving this query, the system will run $\operatorname{Init}\left(\lambda_{1}\right)$ and output $P_{A_{j}}$ 's private/public key pair $\left(S K_{A_{j}}, P K_{A_{j}}\right)$ together with identity $S I D_{A_{j}}$ to $A$.

3) $\mathcal{O}_{3}: \operatorname{Execute}\left(U_{i}, P_{j}\right)$ - Upon receiving this query, $\prod_{U, P}^{i}$ and $\prod_{P, U}^{j}$ will execute protocol as $U_{i}$ and $P_{j}$ in $\prod$, respectively. The exchanged messages between them will be recorded and sent to $A$. Here, we require that both $U_{i}$ 's credential and $P_{j}$ 's private key are not been corrupted by $A$ via $\mathcal{O}_{1}$ and $\mathcal{O}_{2}$ oracles.

4) $\mathcal{O}_{4}: \operatorname{Send}\left(U_{i}, m, f\right)$-This query sends the message $m$ as message flow $f \in\{0,1, \cdots, n\}$ to the user oracle $\prod_{U, P}^{i}$ which simulates a user $U_{i}$, and then, the oracle computes message honestly in $\prod$, and sends responses back to $A$, where $n$ is the total number of messages transmitted in protocol $\Pi$. If a user is the protocol initiator by default, $A$ can also start a new session by asking $\operatorname{Send}\left(U_{i}, \emptyset, 0\right)$, where $\emptyset$ denotes an empty set.

5) $\mathcal{O}_{5}: \operatorname{Send}\left(P_{j}, m, f\right)$-This query sends the message $m$ as message flow $f \in\{0,1, \cdots, n\}$ to the user oracle $\prod_{P, U}^{i}$ which simulates a service provider $P_{j}$, and then, the oracle computes message honestly in $\prod$, and sends responses back to $A$. If a service provider is the protocol initiator by default, $A$ can also start a new session by asking $\operatorname{Send}\left(P_{j}, \emptyset, 0\right)$.

6) $\mathcal{O}_{6}:$ Reveal $\left(\prod, i\right)$-This query models the leakage of session key in session $i$. This query only can be asked when a session key has been shared between a service provider and a user in session $i$.

Remark 3. $\mathcal{O}_{3}$ simulates the real environment for a passive attacker $A$ who can eavesdrop all messages exchanged between $U_{i}$ and $P_{j}$ when executing protocol $\prod$. If $A$ knows $U_{i}$ 's credential $C_{i}$ and $P_{j}$ 's private key $S K_{j}$, oracle $\mathcal{O}_{3}$ is not necessary as $A$ can run protocol $\prod$ by itself on behalf of them. If A knows one of these two secrets but not both, A can run protocol $\prod$ with $U_{i}\left(P_{j}\right)$ whose secret is not released via executing oracle $\mathrm{O}_{4}\left(\mathrm{O}_{5}\right)$.
Remark 4. $\mathcal{O}_{4}$ simulates the real environment for an active attacker $A$ who may obtain a service provider $P_{j}$ 's private key $S K_{j}$, send message $m$ as message flow $f \in\{0,1, \cdots, n\}$ to a target user $U_{i}$ and then get the corresponding response. To answer this oracle, $U_{i}$ will generate his/her response according to the specification of protocol $\prod$ and sends it to A. Notes that if $U_{i}$ did not receive all necessary previous messages that match this message with message flow $f$, this oracle request will be rejected, since it is meaningless in the view point of $U_{i}$. Actually, $\mathcal{O}_{4}$ also provides adversary A oracle access on algorithm UPGen $(\cdot)$ since $\prod_{U, P}^{i}$ will run $U P G e n(\cdot)$ somehow in executing $\prod$. In our construction, UPGen(.) is Schnorr signature generation algorithm. In this case, on the one hand, oracle $\mathcal{O}_{4}$ may be not stronger than the signing oracle in Game-UFCMA reviewed in section IV, since the temporal message $M$, one input of algorithm UPGen (.), may be jointly decided by $U_{i}$ and $A$ (playing the role of one $\left.P_{j}\right)$, rather than just by $A$. So, it may be hard for $A$ to get $U_{i}$ 's user proof for any arbitrary message $M$. On the other hand, adversary A may be not weaker than the forger in GameUFCMA since besides $\mathcal{O}_{4}$ we also offer other oracle queries, which may increase A's ability. We omit a similar remark which applies to $\mathcal{O}_{5}$.

To formally define the soundness and credential privacy, we first discuss the difference between soundness and credential privacy since the majority of existing schemes only consider the credential privacy. The credential privacy requires unforgeability and irrecoverableness. The former guarantees that any PPT adversary $A$ has only a negligible probability for successfully forging a valid credential $C_{t}$ of a target user $U_{t}$ in the credential generation phase, while the latter requires that in user authentication phase, any $A$ can only recover $C_{t}$ with a negligible probability. Soundness is also critical in the user authentication phase as it ensures that any $A$ without a valid credential can only generate a user proof up that passes through user authentication with a negligible probability. The existing studies [16], [12] only focus on if a valid credential can be forged or recovered by attackers, but do not consider if a valid credential is definitely necessary for generating a valid user proof. We shall define these three properties as a single definition (but one for users and one for service providers).

Let $A^{\mathcal{O}}$ denotes an adversary $A$ who has access to all oracle queries in $\mathcal{O}=\left\{\mathcal{O}_{i} \mid i=1,2, \cdots, 6\right\}$ in adversary model; let the credential holder $U_{i}$ with identity $I D_{i}$ and credential $C_{i}$, and the service provider $P_{j}$ with identity $S I D_{j}$ and key pair $\left(S K_{j}, P K_{j}\right)$ are two polynomial-time Turing machines. Let $U_{i}$ and $P_{j}$ interact with each other, and place $A$ between $U_{i}$ and $P_{j} . \epsilon$ denotes a negligible function. We define secure credential based user authentication as follows:

Definition 3. (Secure credential based user authentication (SCUA)) An AKESSO scheme achieves secure credential based user authentication, if any PPT adversary $A$ has a negligible advantage $A d v^{S C U A}\left(A^{\mathcal{O}}\right)$ for creating a valid user proof without holding the corresponding credential. Formally, 
for any $P P T A, A d v^{S C U A}\left(A^{\mathcal{O}}\right) \triangleq \operatorname{Pr}\left[\left(I D_{t}, u p_{t}, M\right) \leftarrow\right.$ $\left.A^{\mathcal{O}} \mid U P \operatorname{Ver}\left(u p_{t}, I D_{t}, P K, M\right)=1\right] \leq \epsilon$ with the following restrictions:

- A has not obtained the credential $C_{t}$ corresponding to $I D_{t}$ via $\mathcal{O}_{1}$ - Register $\left(\prod, U\right)$ oracle; and

- A has not obtained any valid user proof up for message $M$ by asking any oracle in $\mathcal{O}$, in particular $\mathcal{O}_{3}$ and $\mathcal{O}_{4}$.

Similarly, the definition of secure service provider authentication is given as below:

Definition 4. (Secure service provider authentication (SSPA)) An AKESSO scheme achieves secure service provider authentication, if any $P P T$ adversary $A$ has a negligible advantage $\operatorname{Adv} v^{S P A}\left(A^{\mathcal{O}}\right)$ for forging a valid service provider proof without holding the corresponding service provider's private key. Formally, for any $P P T A, A d v^{S S P A}\left(A^{\mathcal{O}}\right) \triangleq \operatorname{Pr}\left[\left(P K_{t}, M^{\prime}, s p p_{t}\right) \leftarrow\right.$ $\left.A^{\mathcal{O}} \mid S P P V \operatorname{er}\left(P K_{t}, M^{\prime}, s p p_{t}\right)=1\right] \leq \epsilon$ with the following restrictions:

- A has not obtained the private key $S K_{t}$ corresponding to $S I D_{t}$ via $\mathcal{O}_{2}$ - Register $\left(\prod, P\right)$ oracle;

- A has not obtained any valid service provider proof $s_{p p}$ for message $M^{\prime}$ by asking any oracle in $\mathcal{O}$, in particular $\mathcal{O}_{3}$ and $\mathcal{O}_{5}$.

Here, we review the freshness and test query Test $\left(\prod, i\right)$ for defining session key security [17]. An adversary can get session keys by asking $\mathcal{O}_{6}$. We say the session key is fresh if and only if the $\mathcal{O}_{6}$ query has not been asked w.r.t. this session. In other words, the fresh session key must be unknown to the adversary. For simplicity, we call the test query as $\mathcal{O}_{7}$, which is a game defined as follows:

- $\mathcal{O}_{7}-\operatorname{Test}\left(\prod, i\right):$ In protocol $\prod$, if $\prod_{U, P}^{i}$ and $\prod_{P, U}^{i}$ accept and share the same fresh session key in session $i$, upon receiving this query, by tossing a coin $b$ the correct session key is returned if $b=0$, otherwise, a random session key is returend. $A$ only can ask this query one time and $A$ needs to output one bit $b^{\prime}$ as the result of guessing $b$. A's advantage in attacking the session key security (SKS) of protocol $\prod$ is defined as $A d v_{\prod}^{S K S}\left(A^{\mathcal{O}^{\prime}}\right)=\left|2 \operatorname{Pr}\left[b^{\prime}=b\right]-1\right|$, where $\mathcal{O}^{\prime}=\mathcal{O} \cup\left\{\mathcal{O}_{7}\right\}$.

Session key security [17] models adversary $A$ 's inability to distinguish the real session key and a random string, as formally defined below.

Definition 5. (Session Key Security) We say an AKESSO satisfies session key security if for any PPT adversary $A$, $A d v_{\prod}^{S K S}\left(A^{\mathcal{O}^{\prime}}\right) \leq \epsilon$, where $\mathcal{O}^{\prime}=\mathcal{O} \cup\left\{\mathcal{O}_{7}\right\}$.

Finally, we can give the definition of secure authenticated key exchange single sign-on scheme.

Definition 6. (Secure Authenticated Key Exchange Single Sign-On Scheme): An AKESSO scheme is called secure if it is correct and satisfies SCUA, SSPA, and session key security.

\section{REVIEW OF CHANG-LEE'S SCHEME}

In 2012, Chang and Lee [12] proposed an improved efficient remote user identification scheme for mobile device users, the scheme employs single sign-on technique, supports session key establishment, and preserves user anonymity. However, the scheme neither provides credential privacy nor soundness due to [6]. In this section, We briefly reviews the Chang-Lee scheme and its drawbacks.

\section{A. Review of the Scheme}

Chang-Lee's $S S O$ scheme consists of three phases: system initialization, registration, and user identification. The details are as follows.

1) System Initialization Phase: The trusted authority TCP determines the RSA key pair $(e, d)$ and a generator $g$, and publishes public parameters.

2) Registration Phase: In this phase, the trusted authority signs an RSA signature $S_{i}=\left(I D_{i} \| h\left(I D_{i}\right)\right)^{d} \bmod N$ to user $U_{i}$ as the credential. For each service provider $P_{j}$, he needs to maintain his own RSA public parameters $\left(I D_{j}, e_{j}, N_{j}\right)$ and private parameter $d_{j}$ similar as TCP.

3) User Identification Phase: In this phase, the session key is $K_{i j}=h\left(I D_{i} \| k_{i j}\right)$, where $k_{i j}$ is the plain DiffieHellman session key. For identifying service providers, an RSA signature scheme has been used; for user authentication, the user need to provide a proof $z=S_{i}^{h\left(K_{i j}\left\|k_{2}\right\| n_{2}\right)}$ $\bmod N$ of credential $S_{i}$, where $k_{2}$ is user's session key material and $n_{2}$ is a random nonce selected by the user. For the purpose of anonymity, the random nonce $n_{3}$ and user identity which used for proof checking has been encrypted via symmetric key encryption scheme with session key $K_{i j}$ (treated as encryption key). The user can pass authentication if $z^{e} \bmod N=S I D_{i}^{h\left(K_{i j}\left\|k_{2}\right\| n_{2}\right)} \bmod N$ dose hold, and the user believes that they are share the same session key if the hashed $n_{3}$ has been received.

\section{B. Review of Attacks}

Two high risky attacks are identified in [6] on ChangLee scheme. The former allows a malicious $P_{j}$ to recover user credential; the latter enables an adversary passing user authentication without a valid credential. They are briefly reviewed below.

1) Credential Recovering Attack: A user $U_{i}$ can pass authentication if he provides the valid proof $z$ of knowledge $C_{i}$. To simplify the discussion, we use $h_{2}$ to denote $h\left(K_{i j}\left\|k_{2}\right\| n_{2}\right)$. So proof $z=S_{i}^{h_{2}}$. It is easy to see that for different proofs in different session, the same credential $S_{i}$ has been encrypted multiple times with different $h_{2}$ but the same modulo $N$. Thus, if a malicious $P_{j}$ has been accessed twice with the same user $U_{i}$, then $P_{j}$ is able to recover $U_{i}$ 's credential $S_{i}$ by using extended Euclidean algorithm. Let us suppose that $\left(z^{\prime}, z^{\prime \prime}\right)$ and $\left(h_{2}^{\prime}, h_{2}^{\prime \prime}\right)$, the proofs and hash values in two different sessions, satisfy $\operatorname{gcd}\left(h_{2}^{\prime}, h_{2}^{\prime \prime}\right)=1$. Then we can find two integers $a$ and $b$ such that $a \cdot h_{2}^{\prime}+b \cdot h_{2}^{\prime \prime}=1$ (in $\mathbb{Z}$ ) due to the extended Euclidean algorithm. Finally, the $P_{j}$ can recover user credential by computing $z^{\prime a} \cdot z^{\prime \prime b}$ 
$\bmod N=S_{i}^{h_{2}^{\prime} \cdot a+h_{2}^{\prime \prime} \cdot b} \bmod N=S_{i}$. The success rate of this attack is about $60 \%$ [6].

2) Impersonation Attack without Credentials: A small RSA public key $e$ has been assumed in this attack, where the "small" requires the binary length of $e$ is much less than the output length of hash function $h$. The rationality of this assumption is given in [6]. In the conversation, if the $h_{2}$ is divisible by $e$, then the adversary computes an integer $b$ such that $h_{2}=$ $e \cdot b$, and calculates proof $z$ by $z=S I D_{i}^{b}$, where $S I D_{i}=$ $I D_{i} \| h\left(I D_{i}\right)$. The verification holds as $S I D_{i}^{h_{2}} \bmod N=$ $S I D_{i}^{b \cdot e} \bmod N=z^{e} \bmod N$. Thus, the adversary can pass user authentication without a valid credential. The success rate of the attack is about $1 / e$ [6].

\section{REVIEW OF SCHNORR SignATURE}

As one of the simplest, shortest, and frequently used signature schemes, Schnorr signature scheme [18], [13] is provably secure in a random oracle model under the assumption that discrete logarithm problem is intractable [19], [20], [21], [15]. We now review Schnorr signature scheme as follows.

Initialisation: The scheme is defined in a cyclic group $G$ of order $q$ with a generator $g \in \mathbb{Z}_{p}^{*}$, were $p$ and $q$ are primes such that $q \mid p-1, q \geq 2^{160}$, and $p \geq 2^{1024}$. A secure hash function $h(\cdot)$ is also selected.

Signature Generation: To sign a message $m$ with private key $x \in \mathbb{Z}_{q}^{*}$, a signer picks a randomness $r \in \mathbb{Z}_{q}^{*}$, and outputs the signature $(a, e, s)$ by computing $a=g^{r} \bmod p$, $e=h(a, m)$, and $s=r+x \cdot e \bmod q$.

Signature Verification: Given a signature $(a, e, s)$ for message $m$ w.r.t. public key $y=g^{x} \bmod p$, the verifier accepts this signature iff $e \equiv h(a, m)$ and $g^{s} \equiv a y^{e} \bmod p$.

Let us denote $\operatorname{Init}(\lambda), \operatorname{SGen}(\cdot)$ and $S \operatorname{Ver}(\cdot)$ the initialisation algorithm, signing algorithm and verification algorithm, respectively. Formally, a signature scheme is called existentially unforgeable if for any PPT forgery algorithm $A$, it can only win the following game, called Game-UFCMA, with a negligible probability [22][23].

- Setup: $(p k, s k) \leftarrow \operatorname{Init}(\lambda)$. Given a security parameter $\lambda$, a public/private key pair is generated by the initialisation algorithm and adversary $A$ is given the public key $p k$.

- Query: $\sigma_{i} \leftarrow \operatorname{SGen}\left(s k, m_{i}\right)$. $A$ runs up to $q$ times to ask the signature signing oracle in an adaptive manner. Each time, the signing oracle will reply a signature $\sigma_{i}$ for each message $m_{i}$ chosen by $A$, where $1 \leq i \leq q$.

- Forge: $A$ outputs a new message and signature pair $\left(m_{j}, \sigma_{j}\right) . A$ wins if

1) $\operatorname{SVer}\left(p k, m_{j}, \sigma_{j}\right)=1$, i.e., $\sigma_{j}$ is a valid signature for message $m_{j}$ under the public key $p k$.

2) $m_{j} \neq m_{i}$, for any $i \in\{1, \cdots, q\}$.

\section{Proposed SCHEME}

This section presents a secure single sign-on scheme with user anonymity for remote user authentication in distributed systems and networks. We use Schnorr signature [18][13] to overcome the drawbacks in Chang-Lee scheme as their user
TABLE I

NOTATIONS USED IN THE SCHEME

\begin{aligned} \hline$T C P &$ The trusted credential provider \\ $P_{j} &$ A service provider \\ $U_{i} &$ A user \\ $S I D_{j} &$ The unique identity of $P_{j} \\ I D_{i} &$ The unique identity of $U_{i} \\ C_{i} &$ The credential of $U_{i} \\ x &$ The long term private key of $T C P \\ y &$ The public key of $T C P \\ E_{k}(M) &$ Symmetric encryption of message $M$ using key $k \\ D_{k}(C) &$ Symmetric decryption of ciphertext $C$ using key $k \\ h(\cdot) &$ A secure hash function \end{aligned}

proof cannot provide soundness and credential privacy while Schnorr signature can. As a proveably unforgeable signature scheme [21], Schnorr signature allows a signer to authenticate him/herself by signing a message without releasing any other useful information about his/her private signing key. In the proposed scheme, the $T C P$ first issues the credential for each user by signing the user's identity $I D_{i}$ according to Schnorr signature. Then, by treating his/her credential as another public/private key pair the user can authenticate him/herself by signing a Schnorr signature on a temporal message generated in the protocol. In contrast, each service provider maintains its own public/private key pair in any secure signature scheme so that it can authenticate itself to users by simply issuing a normal signature. Finally, as does in Chang-Lee scheme [12], the session key is established by running a variant of DiffieHellman key exchange protocol, and the user anonymity is guaranteed by symmetric key encryption. The notations used in the scheme are summarised in Table I.

System Setup Phase: In this phase, TCP initializes his/her public and private parameters as Schnorr signature scheme. Firstly, TCP picks large primes $p$ and $q$ such that $q \mid p-1$, chooses a generator $g$ of large safe prime order $q$ in cyclic group $G$. Then, $T C P$ sets its private key $S K=x$, where $x \in \mathbb{Z}_{q}^{*}$ is a random number, and publishes its public key $P K=y$, where $y=g^{x} \bmod p$.

Registration Phase: In this phase, user asks $T C P$ for registration, then $T C P$ issues a unique identity $I D_{i}$ via $\operatorname{IdGen}\left(R I_{i}\right)$ and signs a Schnorr signature $(a, e, C)$ for user's identity as credential generation algorithm $C G e n\left(I D_{i}, S K\right)$. $C$ is kept secret by user, while $(a, e)$ will be made public. The details are given below.

- User Registration: When a user $U_{i}$ asks for registration, $T C P$ selects a unique identity $I D_{i}$ and generates a credential $C_{i}=(a, e, C)$ for $U_{i}$ by selecting a randomness $r \in \mathbb{Z}_{q}^{*}$ and computing $a=g^{r} \bmod p, e=h\left(a, I D_{i}\right)$, and $C=r+x e \bmod q$. Then, TCP sends identity $I D_{i}$ and credential $C_{i}$ which is Schnorr signature for $I D_{i}$ to user $U_{i}$, where $C$ should be kept as a secret.

- Service Provider Registration: Each $P_{j}$ maintains a public/private key pair $\left(P K_{j}, S K_{j}\right)$ of any secure signature scheme. Here, algorithms SPPGen (.) and SPPVer $(\cdot)$ are identical to the signature generation and verification algorithms respectively. 


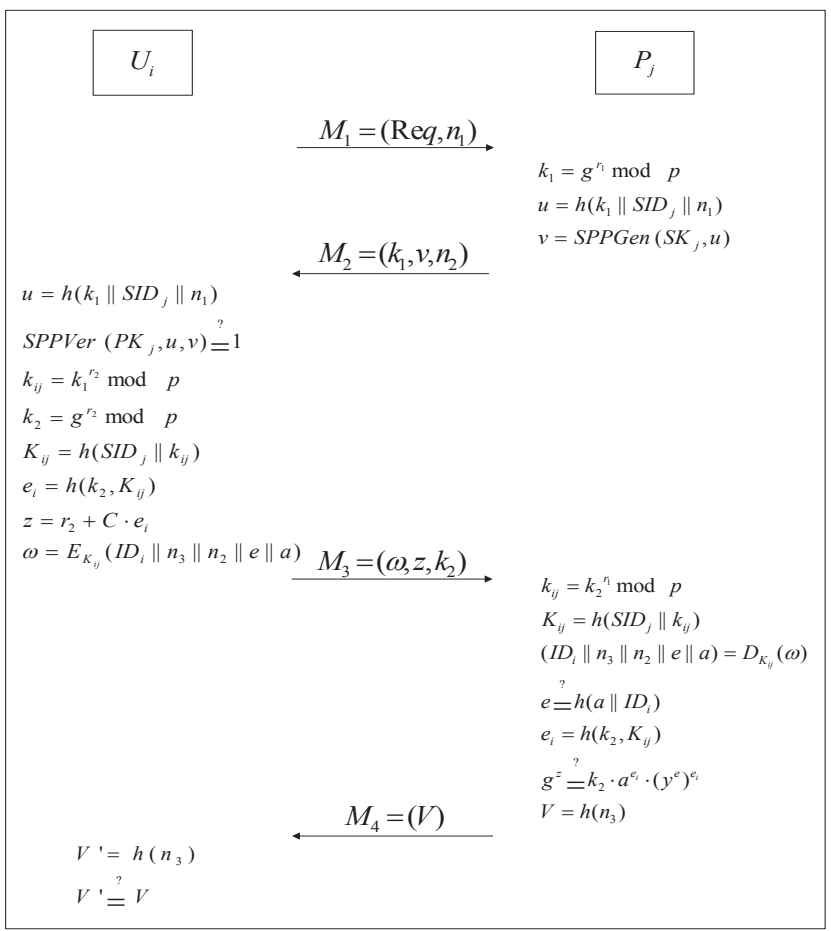

Fig. 1. Participant Identification Phase

Authentication Phase: In this phase, to authenticate him/herself user $U_{i}$ signs a Schnorr signature the newly established session key $K_{i j}$ using credential $C$ the signing key, while $U_{i}$ 's session key material $k_{2}$ is used as the commitment. Note that the corresponding verification key of $C$ is $g^{C}$, which can be recovered by computing $g^{C}=a \cdot y^{e} \bmod p$. For service provider authentication, any provably secure signature scheme can be used to authenticate a service provider in proposed scheme. The session key is established by using modified Diffie-Hellman key exchange scheme which has been formally proved in [12], and the user anonymity and unlinkability are preserved by using symmetric key encryption to encrypt $a, e$, and user's identity $I D_{i}$. The details of this phase are illustrated in Figure 1 and further explained below.

1) User $U_{i}$ chooses a random nonce $n_{1}$ and sends $M_{1}=$ (Req, $\left.n_{1}\right)$ to $P_{j}$, where $R e q$ is a service request.

2) Upon receiving $\left(R e q, n_{1}\right), P_{j}$ picks random number $r_{1} \in \mathbb{Z}_{q}^{*}$, computes its session key material $k_{1}=g^{r_{1}}$ $\bmod p, u=h\left(k_{1}\left\|S I D_{j}\right\| n_{1}\right)$ and signs $u$ to get a signature $v=\operatorname{SPPGen}\left(S K_{j}, u\right)$, and sends $M_{2}=$ $\left(k_{1}, v, n_{2}\right)$ to the user.

3) User $U_{i}$ first computes $u=h\left(k_{1}\left\|S I D_{j}\right\| n_{1}\right)$ and verifies the signature $v$ by checking if $S P P V \operatorname{er}\left(P K_{j}, u, v\right)=1$. If the output is " 0 ", $U_{i}$ terminates the protocol. Otherwise, $U_{i}$ accepts the service provider $P_{j}$ 's authentication, and then selects a random number $r_{2} \in \mathbb{Z}_{q}^{*}$ to compute $k_{2}=g^{r_{2}} \bmod p, k_{i j}=k_{1}^{r_{2}} \bmod p$, and the session key $K_{i j}=h\left(S I D_{j} \| k_{i j}\right)$. After that, $U_{i}$ signs $K_{i j}$ using his/her credential secret $C$ by calculating $e_{i}=h\left(k_{2}, K_{i j}\right), z=r_{2}+C e_{i} \bmod q$ and $\omega=E_{K}\left(I D_{i}\left\|n_{3}\right\| n_{2}\|e\| a\right)$, where $n_{3}$ is a nonce chosen by $U_{i}$. Finally, $U_{i}$ sends $M_{3}=\left(\omega, z, k_{2}\right)$ to service provider $P_{j}$.

4) To verify $z, P_{j}$ first calculates $k_{i j}=k_{2}^{r_{1}} \bmod p$, derives session key $K_{i j}=h\left(S I D_{j} \| k_{i j}\right)$ and decrypt $\omega$ with $K_{i j}$ to recover $I D_{i}\left\|n_{3}\right\| n_{2}\|e\| \mid a$. Then, $P_{j}$ checks if $e=h\left(a \| I D_{i}\right)$. If this does not hold, $P_{j}$ aborts the protocol. Otherwise, the service provider computes $e_{i}=h\left(k_{2}, K_{i j}\right)$ and verifies $z$ by checking if $g^{z}=$ $k_{2} \cdot a^{e_{i}} \cdot\left(y^{e}\right)^{e_{i}} \bmod p$. If this holds, $P_{j}$ accepts $U_{i}$ 's authentication, believes that they have shared the same session key $K_{i j}$, and sends $V=h\left(n_{3}\right)$ as $M_{4}$ to $U_{i}$.

5) User $U_{i}$ computes $V^{\prime}=h\left(n_{3}\right)$ and checks if $V^{\prime}=V$. If this holds, $U_{i}$ believes that he/she has shared the same session key $K_{i j}$ with $P_{j}$.

\section{SECURITY ANALYSIS}

The proposed scheme employs Schnorr signature scheme [18][13] to generate credentials for users, uses modified DiffieHellman key exchange scheme to establish the session key, signs a Schnorr signature on the hashed session key for user authentication, uses any secure signature scheme for server authentication, and takes symmetric key encryption to ensure user anonymity. The secure authenticated key exchange single sign-on (AKESSO) scheme requires secure credential based user authentication $(S C U A)$, secure service provider authentication $(S S P A)$, and secure session key. To prove the security of proposed AKESSO, we will just prove $S C U A$ and $S S P A$ because (1) the proposed scheme only improves parts of key generation, user authentication and service provider authentication in Chang-Lee scheme [12], while the parts of user anonymity and session key establishment have not been modified; and the user anonymity and session key security have been proved in [12] and discussed in [6] without revealing any problems. Now, we start to formally analyse the security of the proposed AKESSO scheme.

Theorem 1. (Correctness) The proposed construction is a correct AKESSO scheme according to Definition 2.

Proof: This can be straightforwardly verified according to Definition 2 given in Section II.

Informally, the proposed AKESSO scheme guarantees $S S P A$ as each service provider employs a secure signature scheme. To prove $S C U A$, we need to show that Definition 3 holds for the proposed AKESSO scheme by assuming the unforgeability of Schnorr signature scheme.

Theorem 2. (Secure Credential based User Authentication) In proposed AKESSO scheme, if there is an PPT adversary A who has a non-negligible advantage $\operatorname{Adv} v^{S C U A}\left(A^{\mathcal{O}}\right)$ as specified in Definition 3, then Schnorr signature scheme is existentially forgeable under UFCMA attacks as defined in Section IV.

Proof: As adversary $A$, with access to all oracles in $\mathcal{O}=\left\{\mathcal{O}_{1}, \cdots, \mathcal{O}_{6}\right\}$, has a non-negligible advantage 
$A d v^{S C U A}\left(A^{\mathcal{O}}\right)$, according to Definition 3 this implies that at least one of the following two cases is true:

- Case (1): With a non-negligible probability $\epsilon_{1}, A^{\mathcal{O}}$ is able to derive a credential $C_{t}$ corresponding to an unregistered target identity $I D_{t}$.

- Case (2): With a non-negligible probability $\epsilon_{2}, A^{\mathcal{O}}$ is able to forge a valid user proof for a new message $M$ w.r.t. a registered target identity $I D_{i}$.

Now, we will prove that if either Case (1) or Case (2) is true, we can construct an algorithm $B$ that is able to break the unforgeability of Schnorr signature, where $B$ runs $A^{\mathcal{O}}$ as a sub-program for fulfilling its purpose.

Case (1). Suppose that $B$ is given a target Schnorr signature scheme with parameter $(p, q, h(\cdot))$ and public key $y=g^{x}$ $\bmod p$, where the private key $x$ is not known to $B$. $B$ 's strategy for wining Game-UFCMA with non-negligible probability is to set up an AKESSO scheme for $A$ and to simulate oracles in $\mathcal{O}$ such that $A$ cannot distinguish the difference between this simulated environment and a real AKESSO scheme. Therefore, $A$ will be able to successfully derive a credential $C_{t}$ for an unregistered identity $I D_{t}$ with probability $\epsilon_{1}$. After that, $B$ can adapt this credential into a forged Schnorr signature for a new message and thus break the unforgeability of Schnorr signature scheme.

Now we describe how $B$ sets up such a simulated AKESSO scheme for $A$. First, $B$ sets $y$ as the public key of $T C P$ and gives $y$ to $B$. Then, each oracle in $\mathcal{O}_{i}(i=1, \cdots, 6)$ can be simulated as follows. To simulate $\mathcal{O}_{1}$ query $B$ can ask its own signing oracle to get a Schnorr signature $C_{i}$ for each identity $I D_{i}$ and then reply $\left(I D_{i}, C_{i}\right)$ to $A$. To simulate $\mathcal{O}_{2}$ query $B$ can simply run $\operatorname{Init}\left(\lambda_{1}\right)$ to get a public/private key pair $\left(S K_{j}, P K_{j}\right)$ for an identity $S I D_{j}$, and then forwards $\left(S I D_{j}, S K_{j}, P K_{j}\right)$ to $A$. As $B$ knows all users' credentials and all service providers's private keys, it can simulate oracles $\mathcal{O}_{3}, \mathcal{O}_{4}, \mathcal{O}_{5}$ and $\mathcal{O}_{6}$ by trivially executing the whole protocol $\prod$, running one move on behalf of a user, running one move on behalf of a service provider, and revealing a session, respectively. Note that as $I D_{t}$ is an unregistered identity in this case, the corresponding user $U_{t}$ will not be involved in any oracle $\mathcal{O}_{i}(i=1, \cdots, 6)$.

It is not difficult to see that the above simulated system is indistinguishable from a real system in the view point of $A$. Hence, $A$ will be able to output a credential $C_{t}$ for target identity $I D_{t}$ with non-negligible probability $\epsilon_{1}$, where $I D_{t}$ is not asked in $\mathcal{O}_{1}$ queries. Therefore, $B$ will simply forward $C_{t}$ as a forged Schnorr signature for message $I D_{t}$. Since $I D_{t}$ is not asked in $\mathcal{O}_{1}$ queries, $A$ does not ask $I D_{t}$ in its signing oracle, i.e., $I D_{t}$ is a new message for $B$. So, $B$ 's forged message-signature pair $\left(I D_{t}, C_{t}\right)$ is valid according to the definition of Game-UFCMA (refer to Section IV). Moreover, $B$ 's success rate is exactly the same as $A$ 's, i.e., $\epsilon_{1}$, which is non-negligible. Consequently, this means that $B$ successfully breaks the unforgeablity of Schnorr signature scheme.

Case (2). This can be proved similarly as Case (1) but $B$ will embed its target Schnorr signature scheme in the user proof generation algorithm for a registered target user $U_{t}$ with identity $I D_{t}$. Details are given as follows.

Suppose that $B$ is given a target Schnorr signature scheme with parameter $(p, q, h(\cdot))$ and public key $y^{\prime}=g^{x^{\prime}} \bmod p$, where the private key $x^{\prime}$ is not known to $B$. First, $B$ sets $y=$ $g^{x} \bmod p$ as the public key of TCP by selecting a random number $x$ as $T C P$ 's private key. For any identity $I D_{i}$ except target identity $I D_{t}$, to answer an $\mathcal{O}_{1}$ query $B$ can directly issue a credential $C_{i}$ for $I D_{i}$ by generating a Schnorr signature for $I D_{i}$ as $B$ knows $T C P$ 's private key $x$. In contrast, $B$ will take $\left(a^{\prime}, e^{\prime}, x^{\prime}\right)$ as the credential $C_{t}$ for target identity $I D_{t}$, where $e^{\prime} \in\{0,1, \cdots, q-1\}$ is a random number, $a^{\prime} \in \mathbb{Z}_{p}^{*}$ is set as $a^{\prime}=y^{\prime} \cdot y^{-e^{\prime}} \bmod p$, and $h\left(a^{\prime}, I D_{t}\right)$ is set as $e^{\prime}$. So, we have $g^{x^{\prime}}=a^{\prime} y^{h\left(e^{\prime}, I D_{t}\right)} \bmod p$. Note that $B$ does not know the value of $x^{\prime}$ and it will be not required to reveal $C_{t}$ to $A$ because $I D_{t}$ is the target identity. In addition, here we can artificially fix the hash value for such a special input $\left(a^{\prime}, I D_{t}\right)$ because Schnorr signature is secure in random oracle where hash function can be viewed as an random function [21]. All other oracles in $\mathcal{O}$ can be simulated as in Case (1), except $A$ asks $\mathcal{O}_{3}$ and $\mathcal{O}_{4}$ queries in which $U_{t}$ with identity $I D_{t}$ is involved. In such scenarios, $B$ can simulate $U_{t}$ to output a valid user proof $u p_{t}$ w.r.t. credential $C_{t}$ by executing the whole protocol $\Pi$ or running one move with necessary help from its own signing oracle w.r.t. public key $y^{\prime}$.

Again, it is not difficult to see that the above simulated system is indistinguishable from a real system in the view point of $A$. Hence, with probability $\epsilon_{2} A$ will be able to output a valid user proof $u p_{t}$ for a message $M$ w.r.t. target identity $I D_{t}$, where $M$ is not asked in $\mathcal{O}_{3}$ and $\mathcal{O}_{4}$ queries. Therefore, $B$ can simply forward $u p_{t}$ as a forged Schnorr signature for message $M$. Since $M$ is not asked in $\mathcal{O}_{3}$ and $\mathcal{O}_{4}$ queries, $A$ does not ask $M$ in its signing oracle, i.e., $M$ is a new message for $B$. So, $B$ 's forged message-signature pair $\left(u p_{t}, M\right)$ is valid according to the definition of Game-UFCMA (refer to Section IV). Moreover, $B$ 's success rate is exactly the same as $A$ 's, i.e., $\epsilon_{2}$, which is non-negligible. Consequently, this means that $B$ successfully breaks the unforgeablity of Schnorr signature scheme.

Remark 5. In Case (1), $A^{\mathcal{O}}$ could directly forge $C_{t}$, recover $C_{t}$ after executing protocol $\prod$ with user $U_{t}$ or eavesdropping the transcripts between $U_{t}$ and some service providers, or derive $C_{t}$ in any other possible way, though $A^{\mathcal{O}}$ is not allowed to obtain $C_{t}$ by trivially asking $\mathcal{O}_{1}$ oracle w.r.t. ID $D_{t}$. Hence, this means that if our AKESSO fails to satisfy the unforgeablity or unrecoverableness of credential, then Schnorr signature is forgeable. Similarly, in Case (2) $A^{\mathcal{O}}$ could directly forge a user proof up $p_{t}$ without credential $C_{t}$, observe and adapts existing user proofs generated by $U_{t}$ into a user proof up $p_{t}$ for a message $M$, or compute upt in any other way, though $A^{\mathcal{O}}$ is not allowed to obtain any user proof for the same message $M$ by trivially asking $\mathcal{O}_{3}$ and $\mathcal{O}_{4}$ oracles w.r.t. ID . Hence, this implies that if our AKESSO fails to satisfy soundness of credential based authentication [6], then Schnorr signature is forgeable. 
As Schnorr signature scheme is proved to be secure under the discrete logarithm assumption [21], Theorem 2 assures that the proposed AKESSO scheme achieves secure credential based user authentication under the discrete logarithm assumption.

Theorem 3. (Secure Service Provider Authentication) In proposed AKESSO, if there is an PPT adversary $A$ who has a non-negligible advantage $\operatorname{Adv} v^{S S P A}\left(A^{\mathcal{O}}\right)$ as specified in Definition 4, then signature signature scheme employed by service providers is existentially forgeable under UFCMA attacks as defined in Section IV.

Proof: Since a service provider proof is directly generated as a normal signature by the corresponding service provider, Theorem 3 can be formally proved as we did for Case (2) in Theorem 1. Note that here we do not need to discuss Case (1) as in Theorem 1, because each service provider is required to register its public/private key pair. Due to space limit, the full proof is omitted.

Theorem 4. According to Definition 6, the proposed AKESSO scheme is secure under the assumption that all digital signatures employed in the scheme are existentially unforgeable against UFCMA attacks as specified in Section IV.

Proof: By Theorem 1, Theorem 2, Theorem 3 and session key security proved in [12], Theorem 4 holds according to Definition 6.

\section{CONCLUSIONS}

Most existing single sign-on schemes suffer from various security issues and are vulnerable to different attacks. In this paper, we first formalized authenticated key exchange single sign-on scheme. Specially, we formally defined secure authentication for both users and service providers as such a treatment has not been studied yet [6]. Moreover, a Schnorr mechanism based SSO scheme has been proposed to overcome the drawbacks of Chang-Lee scheme [12] but keep the same advantages. In this new scheme, to preserve credential generation privacy, the TCP signs a Schnorr signature [18][13] on user identity; and to protect credential privacy and soundness, the user exploits his/her credential as a signing key to sign a Schnorr signature on the hashed session key. In fact, Schnorr signature mechanism [18][13] is more efficient than RSA mechanism which has been employed by Chang-Lee scheme. Thus, the proposed scheme reduces the computation cost, enhances the confidentiality, and preserves soundness and credential privacy.

\section{REFERENCES}

[1] A. C. Weaver and M. W. Condtry, "Distributing Internet Services to The Networks Edge”, IEEE Trans. Ind. Electron., vol. 50, no. 3, pp. 404-411, Jun. 2003.

[2] L. Barolli and F. Xhafa, "JXTA-OVERLAY: A P2P Platform for Distributed, Collaborative and Ubiquitous Computing", IEEE Trans. Ind. Electron., vol. 58, no. 6, pp. 2163-2172, Oct. 2010.

[3] L. Lamport, "Password Authentication with Insecure Communication", Commun. ACM, vol. 24, no. 11, pp. 770-772, Nov. 1981.
[4] F. Bao, R. H. Deng, "Privacy Protection for Transactions of Digital Goods", Proceedings of the Third International Conference on Information and Communications Security (ICICS '01), Springer-Verlag, London, UK, pp. 202-213.

[5] The Open Group, "Security Forum on Single Sign-on", http://www. opengroup.org/security/12-sso.htm.

[6] G. Wang, J. Yu, and Q. Xie, "Security Analysis of A Single SignOn Mechanism for Distributed Computer Networks", IACR Cryptology ePrint Archive, Report 2012/107, http://eprint.iacr.org/2012/107.

[7] W. B. Lee and C. C. Chang, "User Identification and Key Distribution Maintaining Anonymity for Distributed Computer Networks", Computer Systems Science and Engineering, vol. 15, no. 4, pp. 113-116, 2000.

[8] T.-S. Wu and C.-L. Hsu, "Efficient User Identification Scheme with Key Distribution Preserving Anonymity for Distributed Computer Networks", Computers and Security, vol. 23, no. 2, pp. 120-125, 2004.

[9] Y. Yang, S.Wang, F. Bao, J.Wang, and R. H. Deng, "New Efficient User Identification and Key Distribution Scheme Providing Enhanced Security", Computers and Security, vol. 23, no. 8, pp. 697-704, 2004.

[10] K. V. Mangipudi and R. S. Katti, "A Secure Identification and Key Agreement Protocol with User Anonymity (sika)", Computers and Security, vol. 25, no. 6, pp. 420-425, 2006.

[11] C.-L. Hsu and Y.-H. Chuang, "A Novel User Identification Scheme with Key Distribution Preserving User Anonymity for Distributed Computer Networks", Inf. Sci., vol. 179, no. 4, pp. 422-429, 2009.

[12] C.-C. Chang and C.-Y. Lee, "A Secure Single Sign-on Mechanism for Distributed Computer Networks", IEEE Transactions on Industrial Electronics, vol. 59, no. 1, pp. 629-637, 2012.

[13] C.P. Schnorr, "Efficient Signature Generation by Smart Cards", J. Cryptology, vol. 4, no. 3, pp. 161-174, 1991.

[14] S. Goldwasser, S. Micali, and C. Rackoff, "The Knowledge Complexity of Interactive Proof-Systems", SIAM J. Computing, vol. 18, no. 1, pp. 186-208, Feb. 1989.

[15] W. Mao, Modern Cryptography: Theory and Practice, Prentice Hall PTR, 2004.

[16] J. Han, Y. Mu, W. Susilo, and J. Yan, "A Generic Construction of Dynamic Single Sign-on with Strong Security," in Proc. of Secure Comm'10, pp. 181-198, LNICS 50, Springer, 2010.

[17] M. Bellare and P. Rogaway, "Entity Authentication and Key Distribution", CRYPTO, pp. 232-249, 1993.

[18] C.P. Schnorr, "Efficient Identification and Signatures for Smart Cards", CRYPTO ,pp. 239-252, 1989.

[19] M. Bellare and A. Palacio, "GQ and Schnorr Identification Schemes: Proofs of Security against Impersonation under Active and Concurrent Attacks", CRYPTO, pp. 162-177, 2002.

[20] D. Pointcheval, J. Stern, "Security Proofs for Signature Schemes", EUROCRYPT, pp. 387-398, 1996.

[21] D. Pointcheval, J. Stern, "Security Arguments for Digital Signatures and Blind Signatures", J.Cryptology, vol.13, no.3, pp. 361-369, 2000.

[22] S. Goldwasser, S. Micali, and L. Ronald, "A "Paradoxical" Solution to the Signature Problem (Extended Abstract)”, FOCS, pp. 441-448, 1984.

[23] S. Goldwasser, S. Micali, and R. L. Rivest, "A Digital Signature Scheme Secure Against Adaptive Chosen-Message Attacks", SIAM J. Comput., vol. 17, no. 2, pp. 281-308, 1988. 